\title{
Antidepressants are not drugs of abuse or dependence
}

\author{
Frederick J Lichtigfeld, Mark A Gillman
}

\begin{abstract}
Summary
There is a view among both the lay and medical audience that antidepressants are addictive. Noncompliance may arise as a result, with fatal consequences in some cases. In spite of the fact that antidepressants have not proved to be drugs of abuse or dependence, confusion exists in the literature, particularly regarding the definition of the terms misuse and abuse in opioid addicts. Here, antidepressants are used to treat the depressive component of the addictive syndrome and have proved effective. In some instances, however, misuse of antidepressants has been found in methadone-treated addicts, which may be due to an enhancement of the effects of methadone. These effects have led some people to believe, wrongly, that antidepressants are substances of abuse. Our findings, from a review of the literature, show that such use of antidepressants by methadone patients is by definition misuse and not abuse. Further, the known withdrawal symptoms which occasionally follow cessation of antidepressant therapy are not, on their own, an indication of addictive potential. We therefore conclude that antidepressant drugs are not substances of abuse and dependence.
\end{abstract}

Keywords: antidepressants; drug abuse; compliance

South African Brain Research Institute, 6 Campbell Street, Waverly 2090, Johannesburg, South Africa

F J Lichtigfeld

M A Gillman

Accepted 23 April 1998
Prior to the advent of tighter definitions of drug abuse, as initially given in the Diagnostic and Statistical Manual of Mental Disorders, 3rd edn, revised $(D S M-I I I-R)^{1}$ and in a publication from the World Health Organisation (WHO) ${ }^{2}$, drug abuse in particular was regarded as “ . . . the persistent or sporadic self-administration of a drug for non-medical purposes or use which deviates from cultural social patterns .... It is most clearly typified by the wide and indiscriminate use of proprietary preparations, particularly the salicylate analgesics, for a range of minor symptomatic ailments without referral for medical advice. Even drugs administered under medical direction are liable to abuse and practitioners are increasingly aware that patients utilise prescribed medicaments in idiosyncratic ways. The borderlines between drug abuse and drug dependence are blurred .....". ${ }^{3}$

Thus it is clear that before 1987 this loose usage of the term drug abuse had become accepted as being part of the addiction process; this false belief still persists with a resultant decrease in compliance with essential psychotropic medications. ${ }^{45}$ Clearly, what had previously been considered drug abuse was now regarded as either misuse or inappropriate use of drugs, while abuse according to $\mathrm{DSM}-\mathrm{IV}^{6}$ is now much more tightly defined (box 1). All of these criteria must occur for at least a one-year period, and abuse is only diagnosed if the patient does not fulfill further criteria for the diagnosis of substance dependence. Even before 1987, however, drug dependence was definable as a characteristic of drug addiction. ${ }^{3}$

Another cause for confusion is the fact that tricyclic antidepressants cause a cholinergic withdrawal state. ${ }^{78}$ It is still often thought that the existence of a withdrawal state automatically implies that the drug causing the withdrawal is an addictive drug, although this, in itself, is not considered an indication that the substance producing the withdrawal state will cause either abuse or dependence (personal communications, SL Dubowsky, HY Meltzer, 1991). Similarly, the specific serotonin reuptake inhibitors (SSRIs) have also recently been shown to cause only transient and inconsequential withdrawal symptoms (Data on File: Paroxetine and other SSRIs: Smith, Kline and Beecham, 1996). Such withdrawal states, on their own, are classified as Substance-Induced Organic Mental Disorders as defined by DSM-III-R. ${ }^{1}$ However, in DSM-IV the term 'Organic' has been dropped. ${ }^{6}$ Instead, Substance-Related Disorders subsume those conditions which arise as side-effects of many medications. Included as Substance-Related Disorders are Substance-Induced Disorders, some of which are caused by withdrawal from a particular medication. Here again, withdrawal states per se are not regarded as indications of either abuse and/or dependence. ${ }^{6}$ In spite of these clearcut definitions, many people, both lay and medical, still believe that the presence of any withdrawal state is automatically associated with either abuse of and/or dependence on that particular agent. We must therefore stress that withdrawal alone is not an acceptable criterion for substance abuse and dependence.

Drugs of addiction, namely those that cause abuse and dependence, have been shown in all cases to target specifically two defined brain regions. These are the locus coeruleus and the meso-limbic nucleus accumbens. ${ }^{9}$ Furthermore these authors state that "... antidepressants do not produce addictive behaviour, presumably because they do not cause adaptations within brain reward pathways, or if they do, they are unlike the adaptations produced by the addictive drugs." Apart from this, there appears to be a genetic basis for addictive behaviour in that such individuals have functional dopamine deficiency.$^{10}$ As a result they are more likely to attempt to overcome their deficiency by stimulating the reward systems mentioned above. ${ }^{9}$

Other criteria, apart from withdrawal, which must also be present to confirm a diagnosis of drug dependence have been defined by the WHO and are given in box $2 .^{3}$ Antidepressants do not by any stretch of the imagination fulfill any of these additional criteria. ${ }^{11}$ 


\begin{tabular}{|l|}
\hline Definition of drug abuse $^{6}$ \\
\hline - failure to fulfill major role obligations \\
- recurrent use of a substance in \\
situations where it is hazardous \\
- recurrent legal problems resulting \\
from substance use \\
- continued use of a substance despite \\
social or interpersonal problems \\
caused by taking the substance
\end{tabular}

Box 1

\section{WHO criteria required to confirm a diagnosis of drug dependence}

- a desire or compulsive need to continue taking a drug and to obtain it by any means

- a tendency to increase the dose

- a psychological and a physiological dependence on a drug

- a detrimental effect on the individual and on society

Box 2

\section{The cause of the confusion}

Initially, non-dependent drug use was examined as a possible factor that might shed some light on the development of dependence. ${ }^{12}$ This was a forerunner of a category developed in the WHO's International Classification of Diseases (ICD) as Non-dependent Abuse of Drugs, which referred to a patient who had come under medical care because of the maladaptive effect of a drug on which there was no dependence, but which had been taken on the patient's own initiative with detriment to his/her health or social functioning. ${ }^{13}$ On occasions, nondependent abuse of drugs was also secondary to medication prescribed for a psychiatric disorder, eg, antidepressants for a major depressive disorder as defined in ICD-9. ${ }^{13}$ This category was carried over to the ICD-10 classification under the category Abuse of Non-dependence Producing Substances. ${ }^{14} \mathrm{~A}$ wide variety of substances fell into this category, none of which produce dependence, such as antidepressants, that can, however, have harmful effects if misused by the patient. In ICD-10 this category specifically excludes abuse of psychoactive substances that are addictive. ${ }^{14}$ The latter classification continues to use the term abuse ambiguously, by differentiating between non-dependent abuse and abuse of psychoactive substances that are addictive. Because of this usage, the confusion is compounded by obfuscating the precise scientific meaning of abuse, which can easily lead to the assumption that any substance producing abuse must be addictive in nature. It is also apparent that the ICD-10 classification has included a definition of non-dependent abuse which is quite different from DSM-IV. In DSM-IV, non-dependent substance abuse is classified as Substance-Induced Disorder under the main classification of Substance-Related Disorders. ${ }^{6}$ The latter main classification includes side-effects from medications which may also be self-inflicted by excessive dosing. This includes the antidepressants and many other drugs, none of which fulfill the criteria for abuse and dependence as defined in DSM-IV. ${ }^{6}$ The contradiction between these two classification systems is highlighted by a WHO publication that appeared in 1988 , just prior to the publication of ICD-10. ${ }^{2}$ In this publication, the author emphasized that the inappropriate use, underuse or overuse of tricyclic antidepressants and neuroleptics should be regarded as misuse and the term abuse should not be applied to such misuse. ${ }^{2}$ These efforts to reconcile the two conflicting classifications came to nought, and they are both still being used. As a result, there is still considerable confusion regarding this matter. For example, the Addiction Research Foundation in Canada has used the term abuse rather than misuse when categorizing some forms of illegal or dangerous use of a medication resulting in negative clinical consequences when the outcome may even be life-threatening ${ }^{15}$; this viewpoint was carried over and included in the Pharmaceutical Inquiry of Ontario. ${ }^{13}$

In order to avoid confusion in defining the abuse potential of a drug, one must be extremely careful when using the ICD-10 classification to specify under which category the medication falls; for example, the category Non-dependent Abuse would, by definition, exclude all dependence-producing drugs. However, even under these circumstances, it is extremely easy to be confused as to the addictive nature of any psychoactive medication. Thus, substance abuse in both ICD and DSM terms refers to addictive substances which produce dependence. Non-addictive substances are subsumed under Non-dependent Abuse (ICD) and Substance-Related Disorders (DSM-IV). It would seem that the use of the term abuse, even if qualified in ICD, can and has led to the false assumption that substances such as antidepressants could therefore be addictive; a conclusion which is not supported by ICD-10, ${ }^{14}$ DSM-IV,${ }^{6}$ nor current scientific research. ${ }^{9}$ In ICD-10, ${ }^{14}$ a new category has been introduced called 'Harmful use'. This is classified as a pattern of psychoactive substance use which has caused damage to the mental or physical health of the user. This could well replace the Non-dependent Abuse category of ICD-10, thereby limiting the term 'abuse' to addictive drugs alone. Perhaps the next ICD classification will replace the category Non-dependent Abuse of Drugs by the term 'Harmful use of non-dependence-producing drugs'. This would fit in much better with the philosophy of the WHO classification, as in 1964 the WHO expert committee dealing with addiction resolved that drug dependence would be the only term used that could be applied to drug abuse generally. ${ }^{16}$ The same philosophy is still in vogue because the WHO has, by means of ICD-10, drawn attention to drug dependence once again as the crucial element in determining the morbidity associated with substance abuse. ${ }^{17}$ Clearly the term 'abuse' is so loaded with its clearcut relationship with drug dependence and addiction that the term 'non-dependent abuse' has now become a contradiction in terms, resulting in further confusion regarding the classification of psychoactive substances. 


\section{Clinical implications}

A good example of the present confusion regarding the abuse potential of antidepressants, is the recent statement "When antidepressant medication is used inappropriately for the wrong reasons, it often becomes addictive. There are innumerable articles worldwide supporting this." 18 This clearly relates to the 'non-dependent abuse' of antidepressants as clarified in ICD-10. However, the association of non-dependence with abuse in the term 'non-dependent abuse' is so closely associated with the connection of abuse and drug dependence, that it is very easy to make this error. As a result the public is led to believe erroneously that antidepressants are addictive. This was emphasized in a recent, random, UK community survey of 2003 people $^{19} ; 78 \%$ of this sample believed that antidepressants were addictive, while most patients treated with antidepressants in primary care settings abandoned taking their antidepressants prematurely. Fear of dependence is one likely explanation for this phenomenon. These findings have important implications when doctors prescribe antidepressants. Discontinuation symptoms following brief interruptions in compliance may be misinterpreted by patients as symptoms of addiction, which may further compromise compliance and can cause ". . . significant morbidity" ${ }^{20}$ with untoward and sometimes even fatal consequences. "Thus the evidence is substantial that a real mortality risk and a considerable morbidity risk attend the treatment, or rather lack of treatment, of depression."21

Another point that has been raised about the possible abuse potential of antidepressants is the fact that they have been prescribed for methadone maintenance patients. Anecdotal reports of abuse claim that, in combination with methadone, antidepressants produce a feeling of tranquillity or a pleasant 'high'. ${ }^{22}$ The combination of antidepressants with methadone is perhaps a reasonable therapeutic regimen for those who suffer from the dual diagnosis of opioid substance abuse syndrome and major depressive disorder. ${ }^{22}$ Indeed, a specific study was conducted in which patients suffering from primary depression complicated by opioid dependence were treated with antidepressants in conjunction with methadone maintenance. The authors do not give the slightest hint that these patients abused the antidepressants given in the study. ${ }^{23}$ However, a more recent contribution to the debate has mentioned that opioiddependent patients given antidepressants might use them as part of the chaotic drug use pattern of such individuals. ${ }^{24}$

With reference to the feeling of tranquillity experienced by patients taking methadone and antidepressants, ${ }^{22}$ it is interesting to note that methadone in combination with antidepressants enhances pain relief. ${ }^{25}$ This potentiation is by no means an isolated instance; previous studies have shown a similar potentiation between other opioids and antidepressants in clinical and animal studies. ${ }^{26}$ Previously we mentioned that, even if antidepressants caused adaptations in brain reward pathways, these would be unlike those produced by addictive drugs. ${ }^{9}$ However, a variety of treatments, including chronic administration of tricyclics and electroconvulsive therapy, can enhance central dopamine systems, while intracranial self-stimulation via electrodes in the ventral tegmental area is enhanced by chronic administration of antidepressants. ${ }^{27}$ Chronic administration of certain antidepressant drugs increases the locomotor response to $\mathrm{d}$-amphetamine; this response is mediated by the meso-accumbens dopamine projection. ${ }^{27}$ It is therefore possible for antidepressants, under certain conditions, to enhance the actions of certain addictive drugs. For instance, amitriptyline has been used to eliminate the depression often encountered in methadone maintenance programmes. Many of these patients admitted they found a combination of methadone and amitriptyline enabled them to achieve euphoria. As a result, many of them wished to have the dose of amitriptyline increased. Notwithstanding this finding, there was no evidence of any such use of amitriptyline among non drug-abusing patients. In other words it was confined to the methadone maintenance patients. ${ }^{28}$ The euphoria induced by the combination of amitriptyline and methadone may be due to a potentiation of the methadone effect. ${ }^{29} \mathrm{~A}$ more recent study has found that dothiepin can cause similar euphoric effects in methadone maintenance patients. ${ }^{30}$ Antidepressant drugs have no immediate effect on mood in depressed patients, or in healthy volunteers. ${ }^{31}$ It seems that the only documented evidence for the immediate effect of antidepressants on mood, namely euphoria, has occurred when these agents are given in combination with a methadone maintenance programme. Even here, it did not occur in the majority of patients, and one must bear in mind the other drugs taken by these patients (including heroin, cocaine, cannabis, alcohol and nicotine ${ }^{32} 33$, which are clearly addictive on their own account.

The confusion regarding the distinction between misuse and abuse, is further exemplified by the use of these terms interchangeably in the literature. ${ }^{28}{ }^{30}$ 
Fortunately the DSM-IV classification is extremely specific when it comes to defining 'abuse' and 'dependence', so that readers are left in no doubt about the fundamental distinction between 'dependence producing' and 'non-dependence producing' psychoactive substances.

DSM-IV has replaced the classification of Substance-Induced Organic Mental Disorder of DSM-III-R with Other or Unknown Substance-Related Disorders, which include most disorders related to medications and toxins. However, this is included in the Substance-Related Disorders section, which deals not only with drugs of abuse (specified as alcohol, amphetamine, caffeine, cannabis, cocaine, hallucinogens, inhalants, nicotine, opioids, phencyclidine, and sedative-hypnotic anxiolytics) but also with the side-effects of medications, and toxin exposure. Thus, the term 'substance' is applied to a drug of abuse, a medication, or a toxin, including numerous prescribed and over-the counter medications as well as psychoactive substances which do not qualify as drugs of abuse or dependence, eg, antidepressants. The criteria for substance dependence are even more strict than those for abuse, further emphasizing that antidepressants are by definition excluded. Significantly, the antidepressants are not listed in any of the groupings of drugs with abuse and dependence liability.

It may be worth recalling the criteria for substance abuse according to $\mathrm{DSM}-\mathrm{IV}^{6}$ (box 1). All of these criteria must occur for at least a one year period, and abuse is only diagnosed if the patient does not fulfill further criteria for the diagnosis of substance dependence. Based on these strict criteria, no antidepressant would qualify as an agent of abuse. Thus, the euphoria caused in some patients by a combination of methadone, antidepressants, and any other drug, must be subsumed under the category of misuse of the antidepressant. From the clinical evidence ${ }^{2830}$ it appears that although some methadone maintenance patients had misused their antidepressants, there is no evidence that this misuse qualified as abuse, as defined in DSM-IV. Thus, the attitude that has haunted this field for many years is exemplified by the late Max Glatt when he wrote ${ }^{34}$ : "Probably no CNS-affecting drug is quite free from the risk of leading to dependence, particularly in unstable personalities, and in particular in those individuals who have already proven themselves...... predisposed towards the development of dependence in the past." Glatt goes on "...care should be taken to chose those drugs which seem, so far, to have shown relatively less abuse liability; among the modern non-stimulating antidepressant drugs...." His apparent fear of the addictive potential of even the modern antidepressants led him to go further: "In principle, it would of course be better to keep away from CNS-affecting agents altogether in the long-term therapy of such people." viz, substance abusers.

We trust that we have now laid to rest, once and for all, this ghost and therefore conclude that antidepressants are neither substances of dependence nor abuse as defined by DSM-IV or ICD-10.

1 Diagnostic and statistical manual of mental disorders, 3rd edn, revised (DSM-III-R). Washington, DC: American Psychiatry Association, 1987.

2 Marks J. Psychoactive drugs: the present situation In: Ghodse H, Khan I, eds, Psychoactive drugs: Health Organisation, 1988; pp8-21.

3 Gordon AM. Dealing with common forms of drug dependence. In: Priest RG, ed. Psychiatry in medical practice. Plymouth: Macdonald and Evans, 1982; pp 195-238.

4 Gillman MA, Lichtigfeld FJ. Abuse of neuroleptic drugs? Br ₹ Addict 1992;87:123.

5 tic drugs? Br f Addict 1992;87:123. Kendrick T. Prescribing antidepressants in gen-
eral practice (editorial). BMF 1996;313:829-30.

6 Diagnostic and statistical manual of mental disorDiagnostic and statistical manual of mental disor-
ders, 4th edn (DSM-IV). Washington, DC: American Psychiatry Association, 1994; pp 175-272.

7 von Brucke FT, Hornykiewicz O, Sigg EB. The pharmacology of psychotherapeutic drugs. New York: Springer, 1969; pp 33, 110.

8 Appleton WS. Practical clinical psychopharmacology, 3rd edn. Baltimore: Williams \& Wilkins, 1988; p 57.

9 Hyman SE, Nestler EJ. Initiation and adaptation: a paradigm for understanding psy153:151-62.

10 Noble EP. The gene that rewards alcoholism. Sci Am Sci Med 1996;3:52-61.

11 Lichtigfeld FJ, Gillman MA. The possible abuse of and dependence on major tranquilizers and 5-6.

12 Geber BA. Non-dependent drug use: some psychological aspects. In: Steinberg H, ed. Scientific basis of drug dependence. London: Churchill, 1969; pp375-87.

13 International Classification of Diseases (ICD 9) vol 1. Classification of mental and behavioural disorders. Geneva: World Health Organisation, 1977.

14 International Classification of Diseases (ICD 10) vol 1. Classification of mental and behavioural disorders. Geneva: World Health Organisation, 1992.

15 Lowy F (Chairman/President). Prescriptions for health: report of the pharmaceutical inquiry of Ontario, Canada. Ministry of Health, Ontario, Canada; 1990

16 Lewis A. Introduction: definitions and perspectives. In: Steinberg H, ed. Scientific basis of spectives. In: Steinberg H, ed. Scientific basis of
drug dependence. London: Churchill, 1969; pp drug depe

17 Desjarlais R, Eisenberg L, Good B, Kleinman A. In: World mental health. New York: Oxford University Press, 1995; p 88

18 Garson P. Medical feud is far from cured. Mail $\mathcal{E}$ Guardian (S African edn), 1996; Oct 27 Nov 2: 9.

19 Priest RG, Vize C, Roberts A, Roberts M, Tylee A. Lay people's attitudes to treatment of depression: results of opinion poll for Defeat Depression Campaign just before its launch BMF 1996;313:858-9.

20 Haddad P. Discontinuing antidepressants. Fam Med 1997;1:6-7.

21 Steel R. Useful psychotropic drugs. In: Priest RG, ed. Psychiatry in medical practice. Plymouth: Macdonald and Evans, 1982; pp 95-131.

22 Cox TC, Jacobs MR, Lablanc AE, Marshman JA. Drugs and drug abuse. Toronto: Addiction Research Foundation of Canada, 1983; $\mathrm{p} 437$. 23 Kleber HD, Weissman MM, Rounsaville BJ, pramine for depression in addicts. Arch Gen Psychiatry 1983;40:649-53.

24 Robson P. Opiate misusers: are treatments effective? In: Hawton K, Cowen P, eds. Practical problems in clinical psychiatry. Oxford: Oxford University Press, 1992; pp 141-58.

25 Melzack R, Wall PD. The challenge of pain, 2nd edn. London: Penguin, 1991; p 209.

26 Eschalier A. Ardid D, Coudore F. Pharmacological studies of the analgesic effect of antidepressants. Clin Neuropharmacol 1992;15(suppl 1):373A-4A.

27 Fibiger HC, Phillips AG. Role of catecholamine transmitters in brain reward systems: implicatransmitters in brain reward systems: implications for the neurobiology of affect. In: Engel J, Oreland L, eds. Brain reward systems
New York: Raven, 1987; pp 61-74.

28 Cohen MJ, Hanbury R, Stimmel B. Abuse of amitriptyline. fAMA 1978;240:1372-3.

29 Cantor R. Methadone maintenance and amitiptyline. $\mathcal{F A M A}$ 1979;241:2378.

30 Dorman A, Byrne P, Talbot D, O'Connor J. Misuse of dothiepin. BMF 1995;311:1502.

31 Checkley S. Neuroendocrine effects of psychotropic drugs. Bailliere's Clin Endocrinol Metab 1991;5:15-33.

32 Simpson DD, Joe GW, Bracy SA. Six-year follow-up of opioid addicts after admission to treatment. Arch Gen Psychiatry 1982;39: 1318-23.

33 Wheeler SF. Substance abuse during pregnancy. Primary Care: Clin Office Pract 1993;20:191207.

34 Glatt MM. A guide to the treatment of alcoholism and drug dependence. In: Horrobin D, Gunn A, eds. The international handbook of medical science, 2nd edn, Oxford: MTP, 1972; pp 